\title{
Técnica extraperitoneal comparada con IPOM plus: Análisis de costos evitados para optimizar el manejo de la hernia ventral por laparoscopia
}

\author{
Extraperitoneal technique compared to IPOM plus: Analysis of saved costs \\ to optimize the management of ventral hernia by laparoscopy
}

\author{
Juan Pablo Ruíz ${ }^{1} \mathbb{D}$, Arnold José Barrios ${ }^{2} \mathbb{D}$, Neil Valentín Vega ${ }^{3} \mathbb{D}$, Alejandro Lora ${ }^{1}$, \\ Gloria Stella Flórez ${ }^{4}$, Fredy Orlando Mendivelso ${ }^{5} \mathbb{D}$
}

\begin{abstract}
MD, especialista en Cirugía General, Departamento de Cirugía, Clínica Universitaria Colombia, Bogotá, D.C., Colombia.
2 MD, FACS, especialista en Cirugía General; jefe, Departamento Quirúrgico, Clínica Colsanitas, Clínica Reina Sofía, Bogotá, D.C., Colombia.

3 MD, FACS, especialista en Cirugía General; Departamento de Cirugía, Clínica Reina Sofía, Bogotá, D.C., Colombia. Departamento de Cirugía, Universidad de la Sabana, Chía, Colombia.

4 MD, residente de Cirugía General, Fundación Universitaria Sanitas, Bogotá, D.C., Colombia.

5 MD, MPH, MSc, especialista en Cirugía General y Epidemiología; FETP (Field Epidemiology Training Program); director, Centro Global de Medicina Basada e Informada en Evidencia Keralty. Clínica Reina Sofía, Bogotá, D.C., Colombia.
\end{abstract}

\section{Resumen}

Introducción. Analizamos los costos en el reparo extraperitoneal de la hernia ventral por laparoscopia, desde la perspectiva del sistema general de salud de Colombia, con el fin de mostrar los beneficios de dicho abordaje y su impacto económico, al compararlo con la técnica más implementada, el IPOM plus.

Métodos. Se realizó un análisis económico de costo-beneficio, desde la perspectiva del Sistema General de Seguridad Social en Salud (SGSSS) de Colombia, comparando los costos del reparo de hernia ventral con la técnica extraperitoneal, TAPP o TEP, versus el IPOM plus. Se tomaron como costos de referencia lo establecido en el manual tarifario de Instituto de Seguros Sociales. Los datos fueron analizados con Stata V.I5

Resultados. Se recolectó y analizo información de I09 procedimientos; 59 del grupo extraperitoneal TAPP/TEP y 50 del grupo IPOM plus, realizados durante los años 2015 a 20I8, por el grupo de pared abdominal de Clínica Colsanitas, identificando un ahorro del $69,8 \%$ o resultados de costo-beneficio a favor del grupo extraperitoneal.

Discusión. El abordaje extraperitoneal en el reparo de hernia ventral se consideró una estrategia de alto costo-beneficio para el sistema de salud, validado por la experiencia del grupo de pared abdominal de Clínica Colsanitas, al compararla con el abordaje habitual. Teniendo en cuenta que los insumos utilizados para la

Fecha de recibido: 31/06/2019 - Fecha de aceptación: 21/08/2019

Correspondencia: Fredy Orlando Mendivelso, Calle 127 \# 20-78, Clínica Reina Sofía, Bogotá, D.C., Colombia. Teléfono: 3043844102. Correo electrónico: fmendivelso@colsanitas.com

Citar como: Ruíz JP, Barrios AJ, Vega NV, Lora A, Flórez GS, Mendivelso FO. Técnica extraperitoneal comparada con IPOM plus: Análisis de costos evitados para optimizar el manejo de la hernia ventral por laparoscopia. Rev Colomb Cir. 2020;35:422-8. https://doi.org/10.30944/20117582.778

Este es un artículo de acceso abierto bajo una Licencia Creative Commons - BY-NC-ND https://creativecommons.org/licenses/by-ncnd/4.0/deed.es 
disección no cambian, la prótesis utilizada para cada una de las técnicas representa un costo importante a considerar, tanto para el sistema como para las instituciones de salud

Palabras clave: hernia ventral; reconstrucción de pared abdominal; procedimientos quirúrgicos; mallas quirúrgicas; prótesis e implantes; infección de la herida quirúrgica.

\begin{abstract}
Introduction. Costs in the extraperitoneal repair of ventral hernia by laparoscopy were analyzed from the perspective of the general health system of Colombia, in order to show the benefits of this approach and its economic impact, when compared with the most implemented technique, IPOM plus.
\end{abstract}

Methods. A cost-benefit economic analysis was performed from the perspective of the General Social Security System in Health (SGSSS) of Colombia, comparing the costs of ventral hernia repair with the extraperitoneal technique, TAPP or TEP, against IPOM plus. The reference costs were taken as established in the rate manual of the Social Security Institute. Data were analyzed with Stata v.I5.

Results. Information from I09 procedures was collected and analyzed; 59 of the extraperitoneal group TAPP / TEP and 50 of IPOM plus group, carried out during the years 2015 to 2018 , by the abdominal wall group of Clinica Colsanitas, identifying a saving of $69,8 \%$ or cost-benefit results in favor of the extraperitoneal group.

Discussion. The extraperitoneal approach in ventral hernia repair was considered a high cost-benefit strategy for the health system, validated by the experience of the abdominal wall group of Clínica Colsanitas, when compared with the usual approach. Given that the inputs used for dissection do not change, the prosthesis used for each of the techniques represents an important cost to consider, both for the system and health institutions.

Keywords: hernia, ventral; abdominal wall reconstruction; surgical procedures; surgical mesh; prostheses and implants; surgical wound infection.

\section{Introducción}

La presencia de hernia incisional representa un problema creciente en salud pública, debido a su impacto en la calidad de vida, la imagen personal de los pacientes, el requerimiento de manejo quirúrgico y su alta a incidencia, estimada en IO-20 $\%$ tras una laparotomía ${ }^{\mathrm{I}}$ Se calcula que un cuarto de la población mundial presenta una hernia ventral a lo largo de su vida; incremento consistente con lo publicado en la literatura, donde se reporta un aumento directamente proporcional al número de laparotomías realizadas, representando la condición patológica más valorada por los cirujanos ${ }^{2}$.

El abordaje laparoscópico de la hernia ventral fue descrito por primera vez por LeBlanc y Booth, con la implantación de una malla de poli-tetrafluoretileno, adherida a la pared abdominal con el fin de cubrir el defecto herniario ${ }^{3}$. Esta técnica marcó el inicio de una nueva era en el manejo de los defectos herniarios, disminuyendo notablemente la morbilidad de las heridas quirúrgicas en comparación con el abordaje abierto, pero exponiendo a las complicaciones de las prótesis en posición intraperitoneal, dentro de las cuales se encuentran la migración de la malla, el dolor crónico, la infección del sitio operatorio y sus altos costos, lo que representa una limitante para la implementación de esta técnica quirúrgica. Al mismo tiempo, ha generado interés científico para el uso y creación de nuevas técnicas y abordajes, con el fin de lograr una adecuada corrección del defecto, con menor tasa de recurrencia y complicaciones 4 .

En la búsqueda de la técnica adecuada para el reparo de la hernia ventral se han estudiado 
varios puntos importantes, entre ellos el método de fijación, el tipo de la malla, el cierre primario del defecto y la posición de la prótesis; dentro de esta última, los lugares utilizados en la implantación de las mallas son supra-aponeurótica, puente, retro-rectal, preperitoneal e intraperitoneal (denominada intraperitoneal onlay mesh, o IPOM, por sus siglas en inglés) ${ }^{4}$.

Una de las técnicas innovadoras descritas es el reparo extraperitoneal, tanto TAPP (transabdominal preperitoneal) como TEP (total extraperitoneal), inicialmente aplicado para las hernias inguinales y extrapolado al manejo de la hernia ventral, que muestra ciertas ventajas, tales como la posición conveniente de la prótesis, que se encuentra recubierta por el peritoneo, por lo que se ve asociado a una menor tasa de formación de adherencias y, por lo tanto, menos complicaciones asociadas a la malla y menores costos debido a los insumos utilizados, especialmente al requerimiento de una malla separadora de tejidos en la técnica intraperitoneal, con un alto costo en comparación con las mallas estándares 5 .

El objetivo del estudio fue valorar la diferencia entre dos alternativas laparoscópicas usadas en el reparo de la hernia ventral, comparando malla e insumos de fijación, y modelando los costos por ambas alternativas.

\section{Métodos}

Se realizó un análisis económico de costobeneficio desde la perspectiva del sistema de salud colombiano. Los costos fueron definidos según el Manual Tarifario del Instituto de Seguro Social (ISS), el cual contiene la codificación del Código Único de Procedimientos en Salud (CUPS) para procedimientos quirúrgicos, usando como referencia el valor asignado según sitio o región anatómica, tipo de procedimiento, nombre del procedimiento y definición del procedimiento de interés (Reparo de la hernia ventral). Los procedimientos analizados para el caso base corresponden a un estudio retrospectivo de cohorte realizado por el Departamento de Cirugía en dos instituciones de tercer nivel en Bogotá (Clínica Reina Sofía y Clínica Universitaria Colombia).

\section{Población}

Se reclutaron pacientes adultos con diagnóstico de hernia ventral y cuyo reparo fue realizado por técnica extraperitoneal laparoscópica o técnica IPOM plus. Los procedimientos fueron programados y realizados por cirujanos del Departamento de Cirugía de dos instituciones de alta complejidad de la ciudad de Bogotá, D.C. Los datos clínicos, la información del procedimiento quirúrgico y los costos según el manual tarifario ISS, fueron recolectados para cada procedimiento realizado en el periodo de estudio (2015-20I8). Los datos fueron sistematizados en hojas de cálculo para su análisis.

\section{Análisis estadístico}

El análisis de los datos incluyó el cálculo de medidas de frecuencia y de tendencia central. Las variables categóricas se presentan en tablas, mediante el cálculo de porcentajes. La evaluación de la función de distribución de normalidad de las variables de interés se determinó mediante la prueba de Shapiro-Wilks. El análisis económico se realizó a través de un modelo lineal generalizado de costos esperados en el reparo de la hernia ventral, con el uso de predictores independientes, como los costos asociados al uso de mallas y dispositivos de fijación, controlando la construcción del modelo jerárquico por los efectos de la técnica quirúrgica, edad del paciente, índice de masa corporal (IMC), tamaño del defecto y volumen o número de procedimientos promedio en cada centro, mediante la siguiente función:

$$
Y_{i}=f\left(x_{i}^{(1)}, \ldots x_{i}^{(k)}\right)+\xi i
$$

Los costos fueron tomados en pesos colombianos (COP) y se modeló con un horizonte temporal de 5 años.

\section{Resultados}

Se recolectó información de I09 procedimientos (59 TAPP/TEP y 50 IPOM plus) practicados durante el periodo 2015-20I8 (tabla I). En ambos grupos, la mayoría de las cirugías se realizó en mujeres $(62 \%)$ y la presencia de condiciones 
Tabla 1. Características clínicas de la población incluida en el análisis.

\begin{tabular}{lcc}
\hline \multirow{2}{*}{ Variable } & TAPP/TEP & IPOM plus \\
\cline { 2 - 3 } & Frecuencia (\%) & Frecuencia (\%) \\
\hline Genero & $40(67,8)$ & $31(62,0)$ \\
Femenino & $19(32,2)$ & $19(38,0)$ \\
Masculino & & \\
Presencia de comorbilidades & & \\
No & $17(28,8)$ & $28(56,0)$ \\
Si & $42(71,2)$ & $22(44,0)$ \\
Tipo de comorbilidad & & \\
Hipertensión arterial & $18(30,5)$ & - \\
Enfermedad & $12(20,3)$ & - \\
cardiovascular & $10(17,0)$ & $12(24,0)$ \\
Otros & $9(15,2)$ & - \\
Hipotiroidismo & $9(15,2)$ & $5(10,0)$ \\
Cáncer & $6(10,2)$ & $11(22,0)$ \\
Diabetes & $58(13)$ & $61(13)$ \\
Edad en años, media (DE) & $28,2(4,2)$ & $29,4(3,8)$ \\
IMC (kg/m²) & $1(1,6 \%)$ & $4(8 \%)$ \\
Tamaño del defecto (cm²) & $29,2(54,4)$ & $25(48)$ \\
ISO & $1(1.6 \%)$ & $2(4 \%)$ \\
Enterotomía & $1(1.6 \%)$ & $2(4)$ \\
Recidiva & & \\
\hline
\end{tabular}

* DE: Desviación estándar; IMC: índice de masa corporal; ISO: Infección del sitio operatorio

basales de riesgo fue de $44 \%$, especialmente debidas a hipertensión arterial y otras enfermedades cardiovasculares. Los procedimientos fueron realizados en población adulta, con edad promedio de 58 años, y según los valores de referencia del índice de masa corporal (IMC) dados por la Organización Mundial de la Salud (OMS), la mayoría de los pacientes se encontraba en estado de pre-obesidad $\left(\mathrm{IMC}=25\right.$ a $\left.29,9 \mathrm{~kg} / \mathrm{m}^{2}\right)$.

La incidencia de infección en el sitio operatorio (ISO) y de recidivas durante el postoperatorio fue significativamente inferior en el grupo extraperitoneal (TAPP/TEP). Los tamaños del defecto de pared fueron mayores en pacientes llevados a reparo de hernia ventral con técnica extraperitoneal (TAPP/TEP) que los manejados con técnica IPOM PLUS.
Tabla 2. Análisis global de costos evitados en las mallas y dispositivos de fijación comparando las técnicas extraperitoneales versus IPOM plus.

\begin{tabular}{lcc}
\hline \multirow{1}{*}{ Variable } & TAPP/TEP & IPOM plus \\
\cline { 2 - 3 } & $(\mathbf{n = 5 9 )}$ & $\mathbf{( n = 5 0 )}$ \\
\hline Costo de Malla & 18.500 .000 & 126.500 .000 \\
Costo de Fijación & 27.650 .000 & 26.500 .000 \\
Costo total $^{*}$ & 45.150 .000 & 153.000 .000 \\
\hline
\end{tabular}

$\left.{ }^{*}\right)$ Valores en pesos colombianos.

Al comparar y valorar la diferencia entre dos alternativas laparoscópicas (TAPP/TEP versus IPOM plus) en la reparación de la hernia ventral se analizó el promedio del costo de la malla y el costo promedio de la fijación en pesos colombianos (tabla 2), encontrando una diferencia significativa entre las dos técnicas comparadas, con un costo evitado a favor de las técnicas extraperitoneales del 69,8 \% bajo el supuesto de equivalencia y no variación en las condiciones basales en los grupos comparados (figura I).

\section{Discusión}

La patología herniaria representa un área de especial interés para los cirujanos generales, y dentro de este grupo, la corrección de la hernia ventral es un tópico que se busca dominar. La incidencia de hernia ventral primaria se mantiene estática, mientras que la de las hernias incisionales aumenta progresivamente, debido al gran número de laparotomías realizadas durante el siglo $\mathrm{XX}^{6}$; con una tasa reportada de $3-20 \%$ en Estados Unidos 7 .

Para el manejo quirúrgico de las hernias ventrales se han descrito diferentes técnicas y abordajes, las cuales se han depurado con el paso del tiempo ${ }^{8}$, debido a la alta tasa de recurrencia descrita al inicio de la implementación de las mismas, donde el reparo primario puede tener una tasa de recurrencia de $50 \%$ a 3 años y el reparo con malla hasta de $25 \%$ '. Lo anterior ha llevado a la incorporación de nuevas técnicas y métodos, tales como los abordajes laparoscópicos y el uso de diferentes posiciones de las 


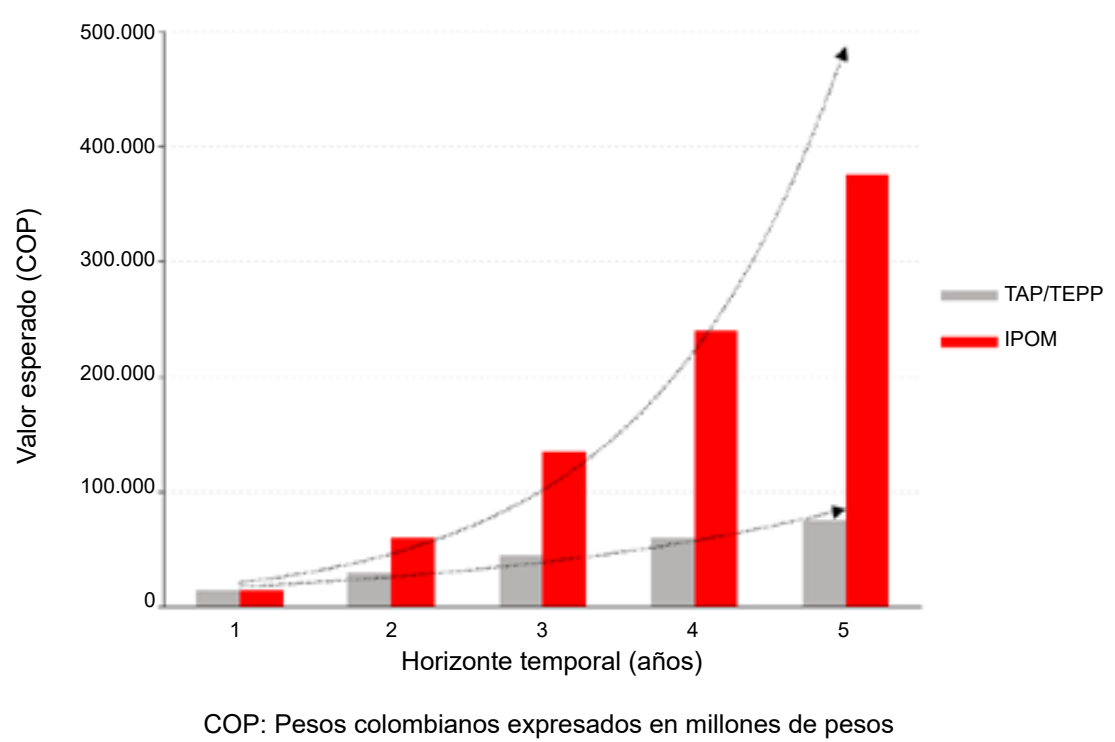

Figura 1. Costos evitados en el reparo de la hernia ventral mediante la estimación del gradiente atribuible al costo esperado en mallas y dispositivos de fijación, controlando por cofactores del paciente, volumen de procedimientos del centro y del procedimiento quirúrgico.

prótesis, con el fin de evitar no solo la recidiva, sino la mayoría de las complicaciones asociadas, $\mathrm{y}$ al mismo tiempo, los costos directos e indirectos de este procedimiento.

El reparo laparoscópico de las hernias ventrales ha aumentado su popularidad con el paso del tiempo, debido a la reducción en la morbilidad y estancia hospitalaria en comparación con el abordaje abierto, sin embargo, no es un abordaje libre de complicaciones ya que se pueden presentar migración de la malla, seroma, adherencias, fístulas, necesidad de retiro de prótesis y recurrencia herniaria secundaria ${ }^{9}$. Con el fin de disminuir la incidencia de dolor crónico postoperatorio (que ocurre entre I,3 y I4,7 \%), el cual está directamente relacionado a la necesidad imperativa de fijación de la prótesis, y las complicaciones asociadas a la malla, que se presentan hasta en $20 \%{ }^{\mathrm{IO}}$, se han descrito nuevas técnicas, dentro de las cuales, las extraperitoneales (tanto TAPP como TEP) han venido ganando terreno, ya que buscan reducir la posibilidad de fístulas intestinales y adherencias, relacionadas a las mallas intraperitoneales ${ }^{\mathrm{II}, \mathrm{I}}$.
En la actualidad, aún no hay consenso sobre cuál puede ser el "estándar de oro" para el reparo de la hernia ventral. Mucho se ha escrito sobre las ventajas de las técnicas intraperitoneales, como IPOM plus, pero las técnicas extraperitoneales juegan ahora un papel importante, en razón a las ventajas descritas.

Para elegir qué técnica utilizar, se deben tener en cuenta, tanto las características del paciente, como las preferencias y habilidades del cirujano. Por otro lado, no se puede despreciar el hecho de que cada decisión tomada influye directamente en los costos para el sistema de salud, entonces, aparece la importancia de los estudios económicos que comparen las técnicas quirúrgicas. En la búsqueda de la literatura, solo se encuentra descrito un análisis de costos, realizado por Moreno-Egea y colaboradores ${ }^{13}$, de características prospectivas, en siete pacientes con hernia de Spiegel llevados a manejo extraperitoneal, sin identificar complicaciones ni recurrencias. Los autores realizan un estudio estadístico comparando TEP e IPOM, identificando un menor costo de esta última, sin embargo, esto se encuentra 
directamente relacionado con el uso del balón, utilizado para crear el espacio extraperitoneal necesario en todos los casos de abordaje TEP.

\section{Conclusiones}

El abordaje extraperitoneal en la cirugía de reparo de hernia ventral, es un manejo innovador, en auge, con múltiples beneficios brindados, no solo por el abordaje mínimamente invasivo, sino por la posición de la malla en la pared abdominal, que ha mostrado resultados adecuados en cuanto a tiempo operatorio, recurrencia y complicaciones ${ }^{14}$. En nuestra experiencia como grupo quirúrgico, el reparo extraperitoneal de la hernia ventral por vía laparoscópica representa una alternativa con múltiples ventajas, que evita la utilización de mallas recubiertas y su fijación invasiva, lo que hacen que sus costos sean menores. Bajo el modelo de comparación, usando datos de dos instituciones de salud con cirugía de alto volumen, se encuentra un costo evitado del $69.8 \%$ con las técnicas extraperitoneales, por lo cual cada vez más, este tipo de técnicas demuestran ser superiores, dados sus beneficios, tanto para el paciente, al evitar complicaciones viscerales de la malla intraperitoneal, el dolor crónico y la infección, como para el sistema de salud, logrando un impacto en los costos en el abordaje de esta patología frecuente.

\section{Cumplimiento de normas éticas}

Consentimiento informado: Este estudio correspondió a una investigación sin riesgo de acuerdo con la Resolución No. 08430 de 1993 del Ministerio de Salud de Colombia, por lo que no se requiere firma de consentimiento informado. El Comité de Ética Institucional y el Comité de investigaciones de las dos instituciones, Fundación Universitaria Sanitas y de Clínica Colsanitas, aprobaron el diseño y la metodología del estudio.

Conflictos de interés. Ninguno declarado por los autores.
Fuente de financiación. Recursos propios de los investigadores.

\section{Referencias}

I. Breuing K, Butler CE, Ferzoco S, Franz M, Hultman CS, Kilbridge JF, et al. Incisional ventral hernias: Review of the literature and recommendations regarding the grading and technique of repair. Surgery. 20IO;I48:544-58. https://doi.org/IO.IOI6/j.surg.20I0.0I.008

2. Holihan JL, Alawadi ZM, Harris JW, Harvin J, Shah SK, Goodenough CJ, et al. Ventral hernia: Patient selection, treatment, and management. Curr Probl Surg. 2016;53:307-54. https://doi.org/I0.I067/j.cpsurg.20I6.06

3. Warren JA, Love M. Incisional hernia repair: Minimally invasive approaches. Surg Clin North Am. 2018;98:537-59. https://doi.org/IO.IOI6/j.suc.2018.0I.008

4. Sanchez LJ, Piccoli M, Ferrari CG, Cocozza E, Cesari M, Maida P, et al. Laparoscopic ventral hernia repair: Results of a two thousand patients prospective multicentric database. Int J Surg. 2018;5I:3I-8. https://doi.org/IO.IoI6/j.ijsu.2018.0I.022

5. Cunningham HB, Weis JJ, Taveras LR, Huerta S. Mesh migration following abdominal hernia repair: a comprehensive review. Hernia. 2019;23:235-43. https://doi.org/IO.I007/sIO029-0I9-0I898-9

6. Sugiyama G, Chivukula S, Chung PJ, Alfonso A. Robot-assisted transabdominal preperitoneal ventral hernia repair. J Soc Laparoendosc Surg. 2015;19:e2015.00092. https://doi.org/I0.4293/JSLS.2015.00092

7. Fernandez-Lobato R, Ruiz de Adana-Belbel JC, Angulo-Morales F, Garcia-Septiem J, Marin-Lucas FJ, Limones-Esteban M. Cost-benefit analysis comparing laparoscopic and open ventral hernia repair. Cir Esp. 20I4;92:553-6o. https://doi.org/IO.IOI6/j.ciresp.2013.04.0I2

8. Daes J, Telem D. The principled approach to ventral hernia repair. Rev Colomb Cir. 2019;34:25-8. https://doi.org/I0.30944/20II7582.94

9. Sauerland S, Walgenbach M, Habermalz B, Seiler CM, Miserez M. Laparoscopic versus open surgical techniques for ventral or incisional hernia repair. Cochrane database Syst Rev. 20II;3:I-6I.

https://doi.org/I0.IO02/I465I858.cdoo778I.pub2

Io. Banerjee A, Beck C, Narula VK, Linn J, Noria S, Zagol B, et al. Laparoscopic ventral hernia repair: does primary repair in addition to placement of mesh decrease recurrence?. Surg Endosc. 20I2;26:1264-8. https://doi.org/IO.IOO7/s00464-OII-2024

II. Prasad P, Tantia O, Patle NM, Khanna S, Sen B. Laparoscopic transabdominal preperitoneal repair of ventral 
hernia: a step towards physiological repair. Indian J Surg. 20II;73:403-8.

https://doi.org/IO.IOO7/sI2262-0II-0366-7

12. Daes J, Daza JC. Separación endoscópica de componentes por abordaje subcutáneo, experiencia inicial. Rev Colomb Cir. 20I4;29:26-32.

13. Moreno-Egea A, Campillo-Soto A, Girela-Baena E, Torralba-Martinez JA, Corral de la Calle M, Agua-
yo-Albasini JL. Traumatic Spigelian hernia. Elective extraperitoneal laparoscopic repair. Cir Esp. 2006;79:6I-3. https://doi.org/I0.IOI6/sooo9-739x(06)708I5

I4. Ruiz J, Barrios A, Lora A, Vega V, Florez G, Mendivelso F. Extraperitoneal laparoscopic ventral hernia repair: one step beyond. Hernia. 2019;23:909-I4.

https://doi.org/IO.IOO7/sI0029-OI9-0I904-O 